EPJ Web of Conferences 73, 08002 (2014)

DOI: $10.1051 /$ epjconf/20147308002

(C) Owned by the authors, published by EDP Sciences, 2014

\title{
The KLOE-2 Project
}

\author{
M. Martini ${ }^{1,2, a}$ on behalf of KLOE/KLOE-2 collaboration $^{\mathrm{b}}$ \\ ${ }^{1}$ Università degli studi Guglielmo Marconi, Roma \\ ${ }^{2}$ INFN, Laboratori Nazionali di Frascati
}

\begin{abstract}
Three new sub-detectors were been installed in the KLOE apparatus of Laboratori Nazionali di Frascati of INFN. New data taking period, pushed by the new optics with crab waisted beam, will start in February 2014. Photon detection is upgraded by means of a small crystal calorimeter, named CCalT, in the very forward direction and of a tungsten-scintillating tile sampling device, named QCalT, instrumenting the low-beta quadrupoles of the accelerator. A new tracking device, the first cylindrical GEM ever built, is inserted to extend tracking capabilities closer to the interaction point. In this paper we present construction details and performances of these detectors that have been installed during summer 2013.
\end{abstract}

\section{Introduction}

From 2000 to 2006, the KLOE experiment has acquired data at the DAФNE $e^{+} e^{-}$collider of the Frascati National Laboratories of INFN. During this period, a record integrated luminosity of $\sim 2.5 \mathrm{fb}^{-1}$ has been collected at a center of mass energy of $1020 \mathrm{MeV}$ corresponding to the mass of the $\phi$ meson, as well as other $\sim 240 \mathrm{pb}^{-1}$ at $1000 \mathrm{MeV}$. This corresponds to an increase of about two orders of magnitude with respect to what collected by previous experiments running the same energy.

During 2008-2009, LNF accelerator division tested the new interaction scheme called "crab-waist". This new optic has been tested by SIDDHARTA collaboration reaching a factor three in peak luminosity respect to the best observed in KLOE during 2005 [1]. Therefore it was natural to propose the implementation of the new scheme also with KLOE, starting a new data taking run with the new collaboration now named KLOE-2.

During KLOE run, the apparatus was composed by two main sub-detectors (see Fig. 1): a very large cylindrical drift chamber, DCH, and a hermetic lead-scintillating fibers electromagnetic calorimeter, EMC, with exceptional timing performance [2-4].

\footnotetext{
ae-mail: matteo.martini@lnf .infn.it
}

${ }^{b}$ D. Babusci, I. Balwierz-Pytko, G. Bencivenni, C. Bloise, F. Bossi, P. Branchini, A. Budano, L. Caldeira Balkestahl, G. Capon, F. Ceradini, P. Ciambrone, F. Curciarello, E. Czerwinski, E. Danè, V. De Leo, E. De Lucia, G. De Robertis, A. De Santis, P. De Simone, A. Di Cicco, A. Di Domenico, C. Di Donato, R. Di Salvo, D. Domenici, O. Erriquez, G. Fanizzi, A. Fantini, G. Felici, S. Fiore, P. Franzini, A. Gajos, P. Gauzzi, G. Giardina, S. Giovannella, E. Graziani, F. Happacher, L. Heijkenskjold, B. Hoistad, M. Jacewicz, T. Johansson, K. Kacprzak, D. Kaminska, A. Kupsc, J. Lee-Franzini, F. Loddo, S. Loffredo, G.Mandaglio, M. Martemianov, M. Martini, M. Mascolo, R. Messi, S. Miscetti, G. Morello, D.Moricciani, P. Moskal, F. Nguyen, A. Palladino, A. Passeri, V. Patera, I. Prado Longhi, A. Ranieri, P. Santangelo, I. Sarra, M. Schioppa, B. Sciascia, M. Silarski, C. Taccini, L. Tortora, G. Venanzoni, W. Wislicki, M. Wolke, J. Zdebik.

This is an Open Access article distributed under the terms of the Creative Commons Attribution License 4.0, which permits unrestricted use, distribution, and reproduction in any medium, provided the original work is properly cited. 


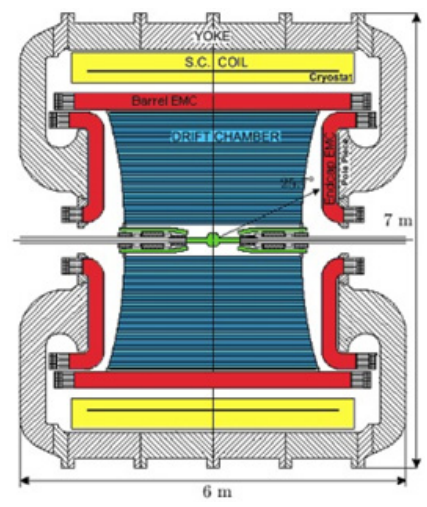

Figure 1. Section of KLOE apparatus with DCH and EMC.

During machine stop, some detector modifications were proposed. In particular, a new LYSO crystals calorimeter, CCalT, has been inserted in front of machine quadrupoles, very close to IP, to intercept very small angles photons from $K_{S}$ and $\eta$ decays [5]. The old quadrupoles calorimeter, QCAL, has been substituted with a new scintillator tiles and tungsten sampling calorimeters, QCalT [6]. Moreover, to increase tracking efficiency we added a new cylindrical GEM tracker, IT, close to IP to cover the region between IP and internal wall of central Drift Chamber [7].

\section{Inner Tracker}

During R\&D phase for KLOE-2, a long work has been dedicated to satisfy space constraints due to existing sub-detectors. The Inner Tracker of KLOE-2 experiment follows a totally new idea of cylindrical GEM detector. In fact, before IT, GEM detectors were only built in a planar geometry.

IT consists of 4 tracking layers at $13 / 15.5 / 18 / 20.5 \mathrm{~cm}$ from IP with diameters of $260 / 310 / 360 / 410 \mathrm{~mm}$. Active length is $70 \mathrm{~cm}$ with $20 \mathrm{~K}$ readout channels in total. Main role of this detector is to improve $\pi^{+} \pi^{-}$vertex resolution to $c \tau_{s} / 3$. Mandatory request for IT is to build a very light detector to avoid kaon regeneration and for this reason material budget is only $2 \% X_{0}$ in the active area. Each layer is a triple-GEM detector, as shown in fig. 2, filled with a gas mixture of $90 \%$ Argon and $10 \%$ Isobutane for a gas gain of $20 \mathrm{~K}$. Spatial resolution with KLOE magnetic field of $0.52 \mathrm{~T}$ are $\sigma_{r \phi}=\sim 250 \mu \mathrm{m}$ and $\sigma_{z}=\sim 400 \mu \mathrm{m}$.

During construction, 3 GEM foils are spliced together with $3 \mathrm{~mm}$ overlap and wrapped on a cylindrical mold. Readout electrodes have been built at CERN TE-MPE-EM as a kapton/copper multilayer flexible circuit. 2D readout has been done with $\mathrm{X}$ longitudinal strips and $\mathrm{V}$ by connection of pads through conductive vias. Measured resolutions are $\sigma_{x}=190 \mu \mathrm{m}, \sigma_{y}=350 \mu \mathrm{m}$.

\section{QcalT}

QcalT are two dodecagonal sampling calorimeters covering the quadrupoles region close to the IP. Each module, 1/12 of one calorimeter, has 16 towers with 5 layers of $3.5 \mathrm{~mm}$ tungsten and 5 scintillator tiles $5 \mathrm{~mm}$ thick (see Fig. 2). Light from scintillator is routed outside via multi-cladding BCF-92 $1 \mathrm{~mm}$ round fibers inserted in a circular groove.

At one side of the calorimeter, fibers are collected and glued into a plastic holder polished with $0.05 \mathrm{~mm}$ planarity and coupled to an aluminum dioxide PCB with 80 SMD SiPM. Each tile is independently coupled to a photodetector ensuring $0.05 \mathrm{~mm}$ clearance in each direction. 

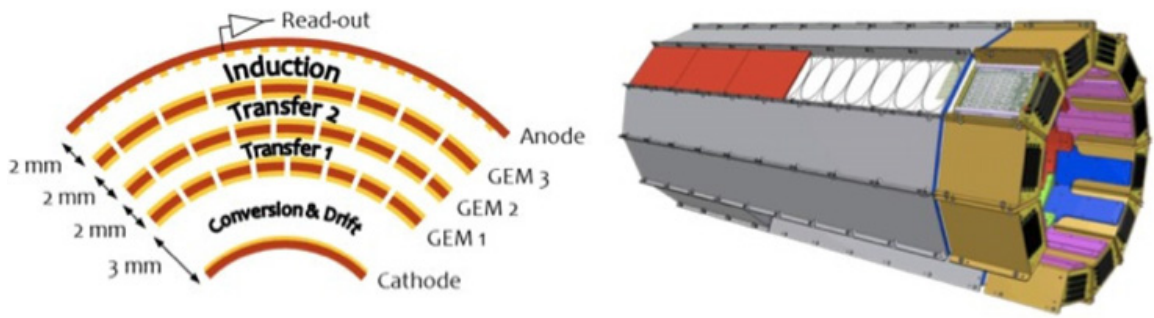

Figure 2. Left: triple GEM for IT. Right: QcalT calorimeter with scintillator tiles.

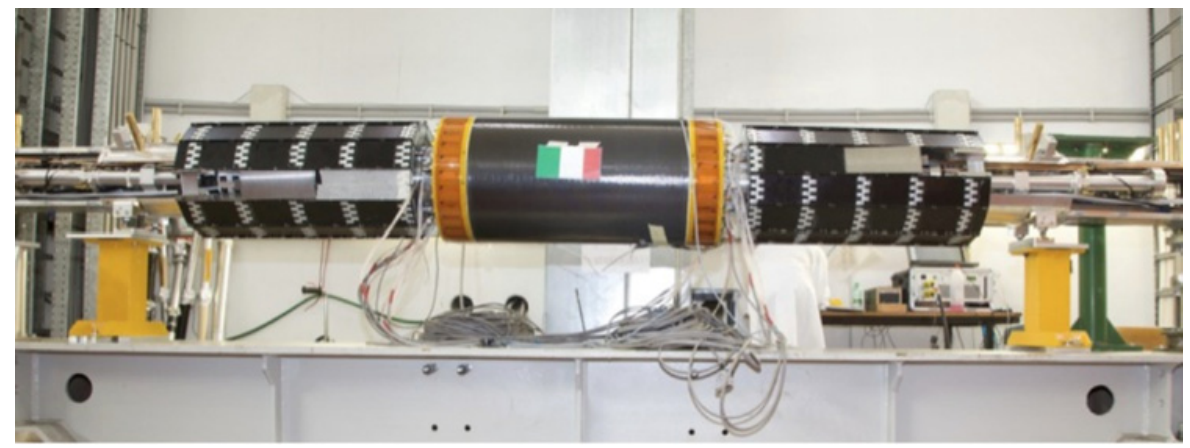

Figure 3. New detectors installed on KLOE-2 beam pipe.

For QcalT calorimeters, $1.2 \mathrm{~mm}$ diameter SiPM have been developed together with the Italian company Advansid. Each PCB is connected to 4 front-end boards each driving 20 channels. These boards house for each channel: HV regulator with $0.1 \%$ precision and $0.01 \%$ stability and a preamplifier x20. During KLOE-2 run, since only timing is measured, calibration of SiPM will be done measuring variation of dark rate when varying both $\mathrm{HV}$ and discriminator threshold.

Presence of QcalT is mandatory to intercept photons coming from $K_{L}$ decays and lost in beam pipe. Increased efficiency on this events implies a strong reduction of background in CP violating events such as $K_{L} \rightarrow \pi^{0} \pi^{0}$. Moreover, high granularity will help on reducing accidental losses.

\section{CCalT}

Photons from $K_{S}$ decays could have very small angle not reaching central electromagnetic calorimeter. To identify these events, we insert a LYSO crystal calorimeter in this region on each side of IP. This solution increases acceptance for central calorimeter from 18 to 10 degrees.

Each CcalT has 48 crystals readout using $3 \times 3 \mathrm{~mm}^{2}$ SiPM. Due to not fully containment of the shower, energy resolution is at the level of $15 \%$ at $100 \mathrm{MeV}$ but with a time resolution of $50 \mathrm{ps}$ (120 ps) for $500 \mathrm{MeV}(100 \mathrm{MeV})$ photons.

\section{New detectors insertion and commissioning}

New detectors have been installed on May 2013 (see Fig. 3) and insertion of the new complete beam pipe inside KLOE has finished on July 2013. 
For beam pipe insertion, some special toolings have been developed to avoid sollecitation on detectors and to route cables ( $\sim 100 \mathrm{Kg}$ per side). This operation was complicated by the small clearence between insertion tooling and DCH internal wall that was only $10 \mathrm{~mm}$.

After beam pipe insertion some long test run have been done for all detectors, including cable connectivity, cooling and cosmic runs.

Moreover, in KLOE-2 apparatus there are also 2 gamma-gamma tagger stations, already inserted during last data taking in 2010, which complete the upgrade realized. A Low Energy Tagger, named LET, is positioned at $1.5 \mathrm{~m}$ from IP and housed into QcalT structure. LET is made by LYSO crystals readout using $3 \times 3 \mathrm{~mm}^{2}$ SiPM and collect photons coming from $e^{+} e^{-}$process with energies between 150 and $350 \mathrm{MeV}$. The High Energy Tagger, or HET, is otherwise positioned $11 \mathrm{~m}$ far from IP and is a scintillator hodoscope inserted in DAФNE beam pipe. HET is realized to collect photon with energy greater than $400 \mathrm{MeV}$.

On August 2013, first electron run has been done by accelerator division and the machine started officially its commissioning on September 2013. Commissioning will finish at the end of January 2014.

Commissioning for new KLOE detectors is in progress including: DAQ integration, slow control and automatic calibration procedure. Between October and December 2013, a large number of cosmic runs has been taken for new detectors to setup readout chain and test performances. Due to not sufficient air flush, QcalT front end electronic reaches a too high temperature (around 35 degrees). During December 2013, a new oil free dedicated air line has been installed decreasing boards temperature down to 20 degrees.

KLOE-2 data taking will officially begin in February 2014.

\section{References}

[1] M. Zobov et al., Phys. Rev. Lett. 104, 174801 (2010)

[2] M. Adinolfi et al., Nucl. Phys. A 663, 1103 (2000)

[3] M. Adinolfi et al., Nucl. Inst. and Meth.. A 482, 364 (2002)

[4] M. Adinolfi et al., Nucl. Inst. and Meth. A 488, 51 (2002)

[5] F. Happacher et al., Nucl. Phys. Proc. Supp. 197, 215 (2009)

[6] M. Cordelli et al., Nucl. Inst. and Meth. A 617, 105 (2010)

[7] A. Balla et al., Nucl. Inst. and Meth. A 628, 194 (2011) 